\title{
Adiponectin inhibits migration and invasion by reversing epithelial-mesenchymal transition in non-small cell lung carcinoma
}

\author{
ENHAI CUI $^{1 *}$, HUIHUI GUO ${ }^{2 *}$, MO SHEN $^{3}$, HUANMING YU $^{2}$, DONGHUA GU $^{2}$, \\ WEI MAO ${ }^{1}$ and XIANG WANG ${ }^{2}$ \\ ${ }^{1}$ Department of Respiratory Medicine, Huzhou Central Hospital; ${ }^{2}$ Center of Clinical Laboratory, \\ The First People's Hospital of Huzhou, Huzhou Teachers College, Huzhou, Zhejiang 313000; \\ ${ }^{3}$ Department of Medical Laboratory, The First Affiliated Hospital of Wenzhou Medical College, \\ Wenzhou, Zhejiang 325000, P.R. China
}

Received December 11, 2017; Accepted June 8, 2018

DOI: $10.3892 /$ or.2018.6523

\begin{abstract}
Adiponectin is the most abundant adipokine in the tumor microenvironment. The role of this protein in tumor progression, however, remains controversial. In the present study, we aimed to investigate the effects of adiponectin on the abilities of migration and invasion in non-small cell lung carcinoma (NSCLC). Using NSCLC cell lines, we examined the effects of adiponectin on cell migration and invasion using Transwell assays. Expression of epithelial-mesenchymal transition markers was examined via microscopy and western blotting. We also performed a knockdown of Twist, AdipoR1 and AdipoR2 in NSCLC cells with siRNAs. The addition of adiponectin to NSCLC cells inhibited both the migration and invasion abilities. Furthermore, we found that NSCLC cells displayed increased epithelial marker expression and downregulation of mesenchymal marker expression following adiponectin administration. Twist AdipoR1 and AdipoR2 knockdown reversed the inhibitory effects of adiponectin on migration and invasion in NSCLC and epithelial-mesenchymal transition. Exogenous adiponectin significantly impaired the
\end{abstract}

Correspondence to: Dr Xiang Wang, Center of Clinical Laboratory, The First People's Hospital of Huzhou, Huzhou Teachers College, Huzhou, Zhejiang 313000, P.R. China

E-mail: xiangw2017@126.com

*Contributed equally

Abbreviations: NSCLC, non-small cell lung cancer; EMT, epithelial-mesenchymal transition; EGFR-TKIs, epidermal growth factor receptor tyrosine kinase inhibitors; MMTV, mouse mammary tumor virus; PyMT, polyoma middle $\mathrm{T}$ antigen; S1P, sphingosine-1-phosphate; DCs, dendritic cells; TAMs, tumor associated macrophages

Key words: adipokine, lung carcinoma, epithelial-mesenchymal transition, cancer therapy migratory and invasive capacities of NSCLC cells through reversal of EMT, suggesting that adiponectin may be a novel promising therapeutic approach against NSCLC.

\section{Introduction}

Lung cancer is a highly prevalent and malignant carcinoma, and with a 5 -year survival rate of less than $20 \%$, it is the leading cause of cancer-related mortality worldwide (1). Recently, a rapid increase in the rate of new cases of lung cancer has been observed in developing countries, in contrast to patterns observed in the developed world. Data from the National Central Cancer Registry of China revealed that lung cancer accounts for the greatest number of cancer-related deaths and exhibits an increasing incidence and mortality trend in China (2). Non-small cell lung cancer (NSCLC) accounts for approximately $80-85 \%$ of lung cancer cases, and displays an aggressive phenotype with almost half of the patients presenting with metastases (3). Metastasis is the primary contributing factor to NSCLC-related mortality. In theory, early diagnosis through screening and $\mathrm{R}_{0}$ resection would effectively prevent metastasis in NSCLC patients. Furthermore, comprehensive evidence has confirmed that early diagnosis of lung cancer through screening is an effective method for reducing mortality (4). Despite the rapid progress in diagnostic techniques such as imaging, and the identification of cancer biomarkers, the rate of successful detection of NSCLC at an early stage has not improved, and the majority of cases present as locally advanced or metastatic disease at the time of diagnosis (5). An understanding of the underlying mechanisms involved in NSCLC metastasis, and the identification of specific targets contributing to this process, contributed to the development of therapeutics to inhibit metastasis and improved the response to antitumor therapies and survival outcomes.

The decoding of the lung cancer genome has led to therapeutic targeting of key gene mutations driving cancer initiation and progression, an approach successfully applied to clinical practice and demonstrating a significant response $(6,7)$. Aside 
from contributing to genetic mutations, various stromal cells and cellular components from tumor microenvironments also contribute to NSCLC development. Cancer-associated fibroblasts display upregulated glucose-regulated protein 78 expression resulting in promotion of NSCLC cell invasion (8). Bertolini et al (9) revealed that signals from the tumor microenvironment elicited epithelial-mesenchymal transition (EMT) and promoted expansion of $\mathrm{CD} 133^{+} / \mathrm{CXCR} 4^{+}$cancer-initiating cells sustaining lung cancer dissemination. EMT not only drives NSCLC migration and invasion, but also promotes drug resistance (10). These data suggest that establishing the role of the tumor microenvironment in the promotion of tumor progression would contribute to the development of novel therapies and improve outcomes with current targeted therapies.

In addition to contributing to intrinsic tumor properties, components from the tumor microenvironment also play an important role in tumor development and immune escape. The adipokine adiponectin, which displays anti-inflammatory activity, is amongst a number of abundant components in the tumor microenvironment, and is specifically released by adipocytes $(11,12)$. Adipose tissue is not only considered as a storage organ, but also as an important endocrine organ that releases various proteins that constitute the tumor microenvironment. Previously data has suggested that adiponectin modulates cell apoptosis and proliferation to limit tumor progression via the heptahelical transmembrane adiponectin receptors 1 (AdipoR1) and 2 (AdipoR2) $(13,14)$. Other studies have challenged the antitumor role of adiponectin by demonstrating that high adiponectin levels appear to favor tumor metastasis and proliferation through activation of the MAPK and NF- $x$ B pathways (15). Epidermal growth factor receptor tyrosine kinase inhibitors (EGFR-TKIs) are an effective treatment for NSCLC patients at an advanced stage. Notably, upregulated adiponectin levels were observed in NSCLC patients treated with EGFR-TKIs, suggesting that adiponectin may promote EGFR-TKI resistance (16). To clarify the effects of adiponectin on migration and invasion in NSCLC and examine the mechanisms involved in this process, NSCLC cell lines were treated with adiponectin. The results of the study confirmed that adiponectin is an important negative regulator of NSCLC migration and invasion through inhibition of the EMT process.

\section{Materials and methods}

Cell lines, reagents and antibodies. Three human NSCLC cell lines (NCI-H1299, HCC827 and A549) were purchased from the Cell Bank of Type Culture Collection of the Chinese Academy of Sciences, Shanghai Institute of Cell Biology, and were cultured in Dulbecco's modified Eagle's medium (DMEM; Gibco; Thermo Fisher Scientific, Inc., Waltham, MA, USA) with $10 \%$ fetal bovine serum (FBS; Gibco; Thermo Fisher Scientific) and $100 \mathrm{U} / \mathrm{ml}$ penicillin and streptomycin in a humidified incubator of $5 \% \mathrm{CO}_{2}$ at $37^{\circ} \mathrm{C}$.

Adiponectin and DAPI were purchased from Sigma-Aldrich (Merck KGaA, Darmstadt, Germany). Antibodies used in the present study included: An EMT antibody kit (cat. no. 9782), $\beta$-actin (dilution 1:1,000; cat. nos. 4970 and 3700), goat anti-rabbit HRP (dilution 1:2,000; cat. no. 7074) and goat anti-mouse HRP(dilution 1:2,000; cat. no. 7076)(Cell Signaling
Technology, Inc., Beverly, MA, USA), anti-E-cadherin (dilution 1:2,000; cat. no. ab76055) and anti-vimentin (dilution 1:2,000; cat. no. ab92547) (Abcam, Cambridge, MA, USA).

Small interfering RNA transfection. One hundred thousand NCI-H1299, HCC827 or A549 cells were plated in 6-well plates in $2 \mathrm{ml}$ of culture medium prior to transfection. A total concentration of $50 \mathrm{nM}$ of siRNAs targeting specified genes or a scrambled control were transfected into monolayer cells at $20-30 \%$ confluency using Lipofectamine 2000 (Invitrogen; Thermo Fisher Scientific, Inc.) ransfection reagent according to the manufacturer's instructions. Transfected cells at 80-90\% confluency were harvested for further studies after 48-72 h.

Cell migration and invasion assays. For the wound healing assay, monolayer NSCLC cells were scratched and cultured with adiponectin or phosphate-buffered saline (PBS). Migrated distances were measured at 0, 24 and $48 \mathrm{~h}$ after the scratch for NCI-H1299, HCC827 and A549 cells. For the migration assays, $2 \times 10^{5}$ NSCLC cells were suspended in $100 \mu \mathrm{l}$ serum-free medium and added into the upper chamber without a coated membrane. For the invasion assays, $2 \times 10^{5}$ NSCLC cells in serum-free medium were placed in the upper chamber coated with a Matrigel membrane. In both assays, $500 \mu \mathrm{l}$ of culture medium with $10 \%$ FBS was placed in the lower chamber. Cells were incubated for $24 \mathrm{~h}$. The cells on the upper side of membrane were removed and those on the reverse side of membrane were fixed with $95 \%$ methanol for 20 min and then stained with $0.4 \%$ crystal violet and counted using an inverted light microscope (Olympus IX71; Olympus Corp., Tokyo, Japan) at x200 magnification.

Protein extraction and western blot analysis. Cells were lysed in RIPA buffer supplemented with a protease inhibitor cocktail for $2 \mathrm{~h}$ and then centrifuged at $24,100 \times \mathrm{g}$ for $10 \mathrm{~min}$ at $4^{\circ} \mathrm{C}$ and supernatants collected. Protein concentrations were assessed using a BCA protein assay kit. A total of $20 \mu \mathrm{g}$ of protein was loaded into each lane and separated by SDS-PAGE on a $10 \%$ gel followed by electrophoretic transfer to a $0.45 \mu \mathrm{m}$ polyvinylidene fluoride (PVDF) membrane. The membrane was blocked with 5\% non-fat milk in Tris-buffered saline comprised of $0.05 \%$ Tween-20 (TBST) for $1 \mathrm{~h}$ at room temperature. The membrane was washed three times with TBST and incubated with the primary antibodies [anti-E-cadherin (dilution 1:2,000; cat. no. ab76055) and anti-vimentin (dilution 1:2,000; cat. no. ab92547)] overnight at $4^{\circ} \mathrm{C}$. The membrane was then washing three times in TBST, and incubated with the secondary antibody goat anti-rabbit HRP (dilution 1:2,000; cat. no. 7074) and goat anti-mouse HRP (dilution 1:2,000; cat. no. 7076) for $1 \mathrm{~h}$ at room temperature. Visualization was performed using SuperSignal West Pico Chemiluminescent Substrate (Thermo Fisher Scientific, Inc.) and analyzed using Image Lab 5.0 software (Bio-Rad Laboratories, Inc., Hercules, CA, USA).

Confocal microscopy. Cells treated with adiponectin or controls were washed with PBS and fixed with 4\% paraformaldehyde. After blocking with 5\% BSA for $30 \mathrm{~min}$ at room temperature, the cells were incubated with anti-E-cadherin and anti-vimentin antibodies overnight in the dark at $4{ }^{\circ} \mathrm{C}$. 
A
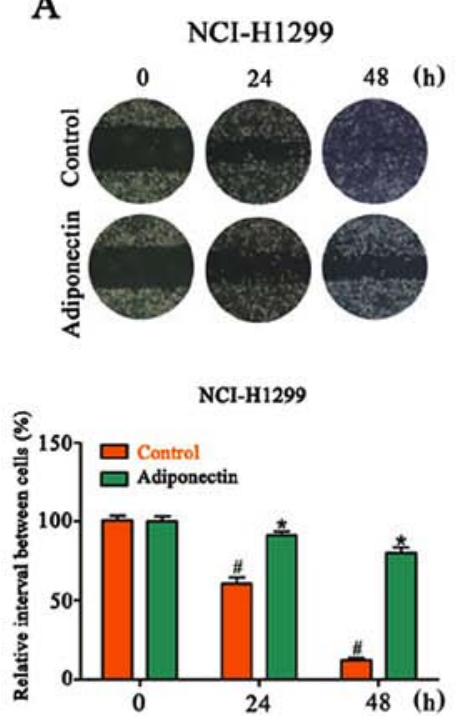

B
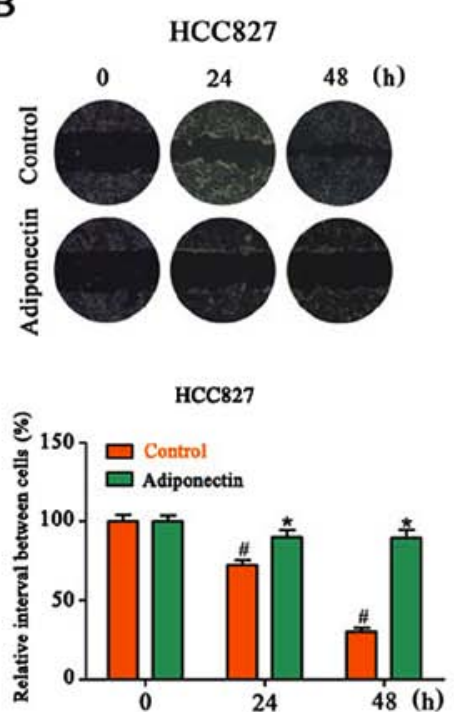

C
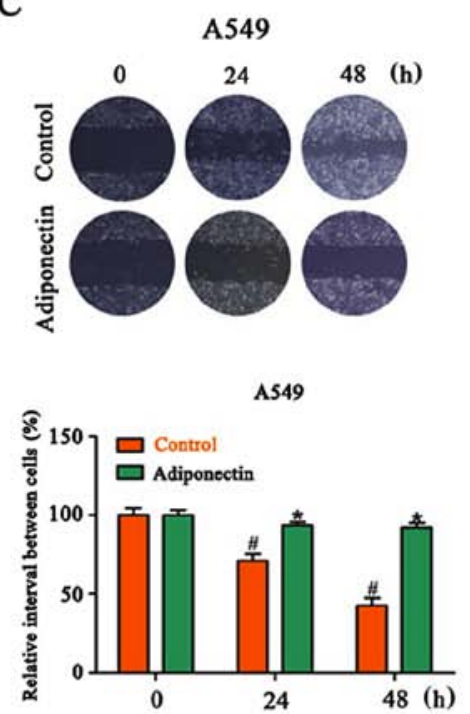

D
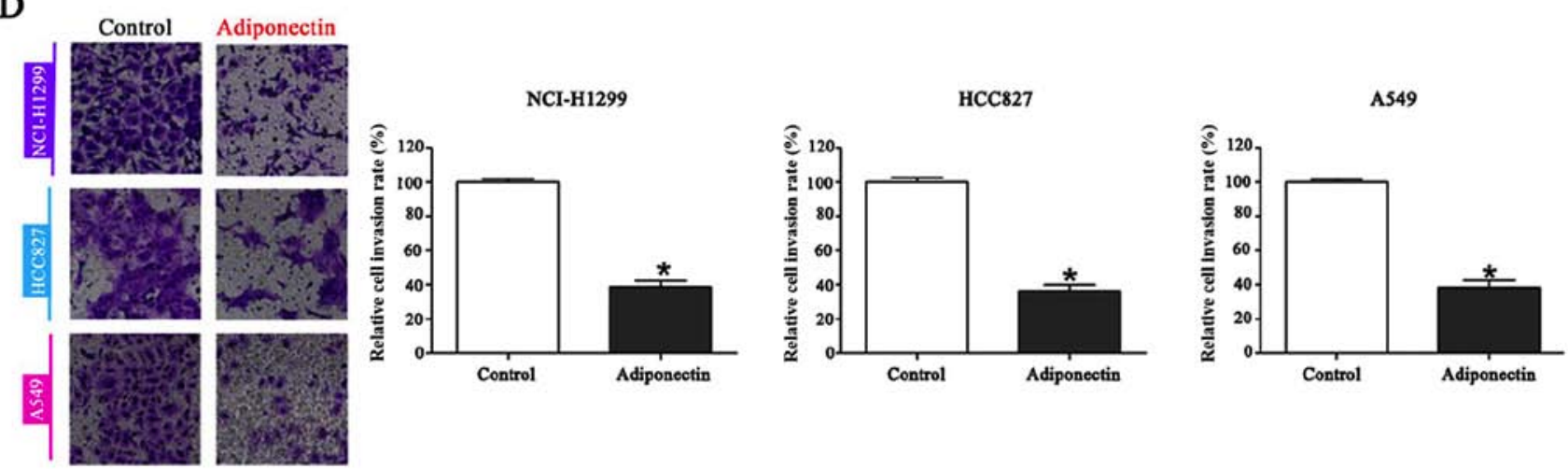

Figure 1. The addition of adiponectin significantly delays healing of an artificial wound in NSCLC cells lines as compared to controls. Wound healing in (A) NCI-H1299, (B) HCC827 and (C) A549 cells at 24 and $48 \mathrm{~h}$ after wounding. (D) Similar results were obtained in Transwell assays, where adiponectin decreased the number of invading cells. ${ }^{*} \mathrm{P}<0.05$ vs. the control $(0 \mathrm{~h}) ;{ }^{*} \mathrm{P}<0.05$ vs. adiponectin $(0 \mathrm{~h}) . \mathrm{n}=3$ (each experiment).

After several washes, the corresponding secondary antibodies conjugated to FITC or $\mathrm{Cy} 3$ were used to detect primary antibodies after incubation for $2 \mathrm{~h}$ at room temperature. The signals were measured with an inverted fluorescence microscope system (Olympus Corp.).

Statistical analysis. Experimental data were presented as the means \pm standard deviations (SD). Statistical analysis was performed using one-way analysis of variance (ANOVA) followed by Tukey's post hoc test and Student's t-test was conducted for comparison between two groups. All data analysis was performed using SPSS software (version 19.0; IBM Corp., Armonk, NY, USA). A value of $\mathrm{P}<0.05$ was considered to indicate a statistically significant result.

\section{Results}

Adiponectin inhibits migration and invasion of NSCLC cell lines. To establish whether increased adiponectin levels in the tumor microenvironment would impact the migration of NSCLC cells, a wound healing assay was performed. Compared with the controls, adiponectin-treated cells demonstrated significantly delayed healing of an artificial wound after 24 and $48 \mathrm{~h}$ (Fig. 1A-C). Since cell invasion is a crucial step in tumor metastasis, an invasion assay was conducted following adiponectin treatment. As expected, NSCLC cell lines treated with adiponectin presented a significant decrease in invasiveness. In the adiponectin group, the number of cells on the reverse side of the Transwell chamber was less than half of the control group (Fig. 1D).

Twist knockdown inhibits NSCLC migration and invasion. E-cadherin and vimentin are biomarkers of EMT. Western blot analysis revealed that the basal levels of expression of E-cadherin were high in HCC827 cells, moderate in A549 cells and low in NCI-H1299 cells. Basal levels of vimentin expression were high in NCI-H1299 cells, moderate in HCC827 cells and low in A549 cells (Fig. 2A). These results were further confirmed by confocal microscopy (Fig. 2B). A siRNA targeting Twist was transfected into NSCLC cells lines to reduce EMT, and resulted in significant inhibition of migration (Fig. 2C-E). Furthermore, Twist knockdown inhibited the invasiveness of NSCLC cells compared with the controls (Fig. 2F-I). 
A

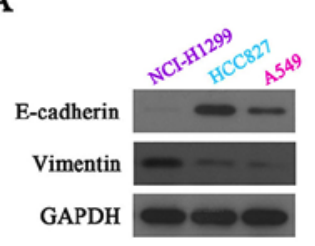

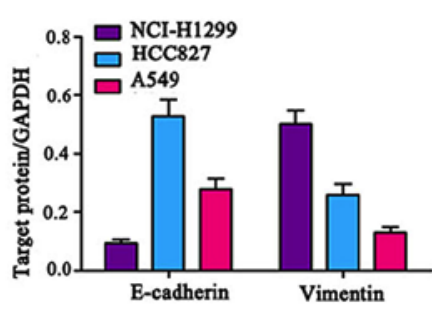

B

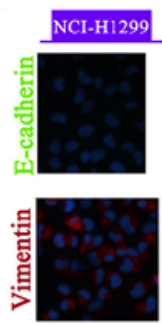

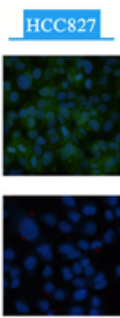

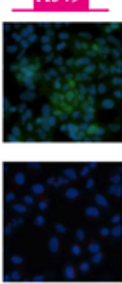

C

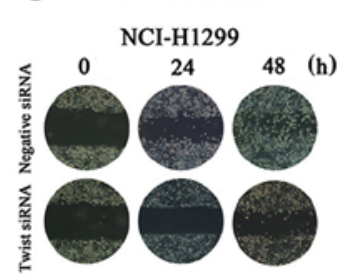

E

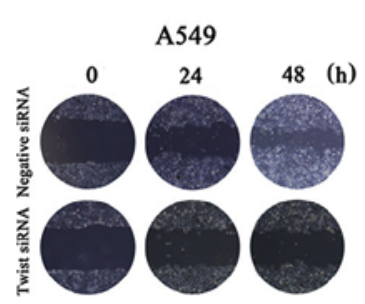

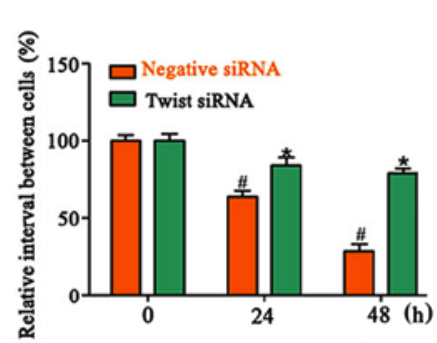

D

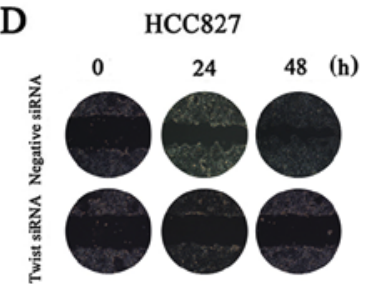

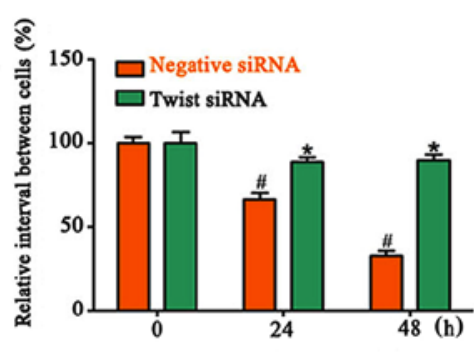

F

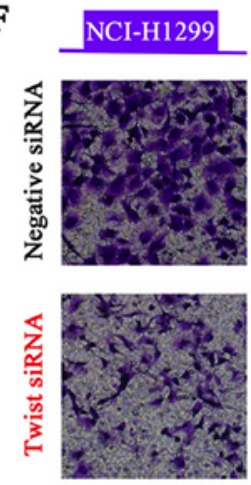

HCC 827
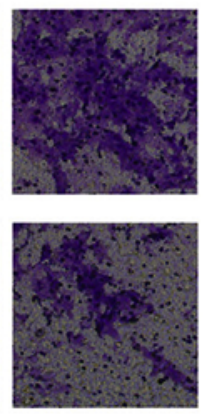

\section{A549}
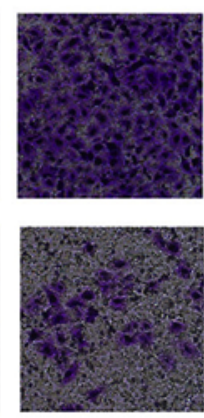

G

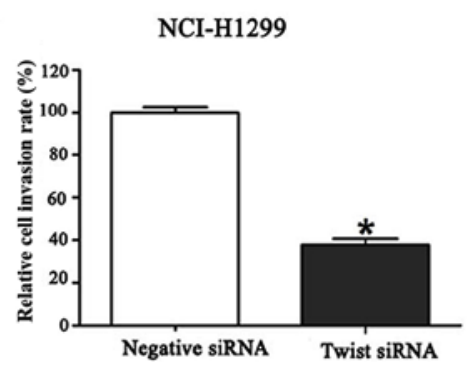

H

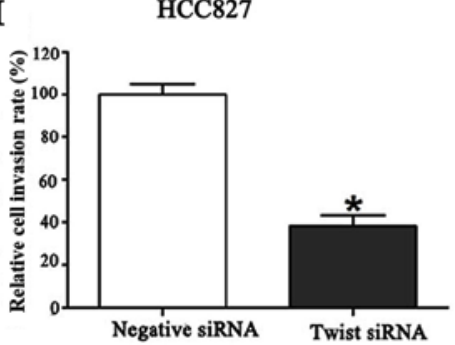

I

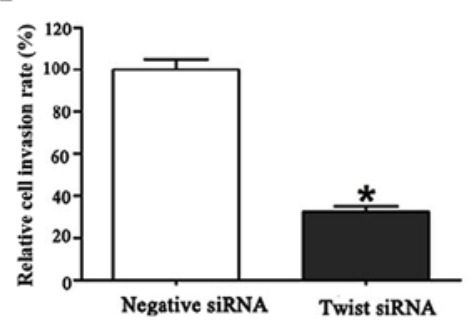

Figure 2. Knockdown of Twist inhibits the migration and invasion of NSCLC cells. (A) Western blotting demonstrating that basal levels of E-cadherin expression were high in HCC827, moderate in A549 and low in NCI-H1299 cells while basal levels of vimentin expression were high in NCI-H1299, moderate in HCC827 and low in A549 cells, $n=3$ (each experiment). (B) Confocal microscopy images confirming the expression levels, $n=3$ (each experiment). Twist knockdown results in significant inhibition of $(\mathrm{C}-\mathrm{E})$ migration $\left[{ }^{\#} \mathrm{P}<0.05\right.$ vs. the control $(0 \mathrm{~h}) ;{ }^{*} \mathrm{P}<0.05$ vs. adiponectin $\left.(0 \mathrm{~h})\right]$ and $(\mathrm{F}-\mathrm{I})$ invasion $\left({ }^{*} \mathrm{P}<0.05\right)$ compared with the controls, $\mathrm{n}=3$ (each experiment).

Adiponectin reverses EMT in NSCLC cell lines. To explore whether adiponectin administration alters the EMT process in NSCLC cells lines, we performed western blotting and confocal microscopy assays to analyze EMT marker expression. The results of western blotting revealed that adiponectin administration led to upregulation of E-cadherin and downregulation of vimentin expression in the three NSCLC cell lines (Fig. 3A-D). Consistent with the western blotting results, the changes in EMT marker expression were visually confirmed by confocal microscopy (Fig. 3E). These results revealed that adiponectin can impair NSCLC cell migration and invasion by reversing EMT.
Twist knockdown eliminates the inhibitory effects of adiponectin on migration and invasion. To further investigate whether reversing EMT is the underlying mechanism involved in the adiponectin-mediated reduction of NSCLC cell migration and invasion, Twist-siRNA-transfected cells were treated with adiponectin. The wound healing assay indicated that Twist knockdown completely eliminated the inhibitory effects of adiponectin on NSCLC cell migration (Fig. 4A-C) and invasion (Fig. 4D). The interaction efficiency of Twist was detected by western blot analysis (Fig. 4E).

AdipoR1 and AdipoR 2 mediate adiponectin function. Previously published studies indicated that the adiponectin 

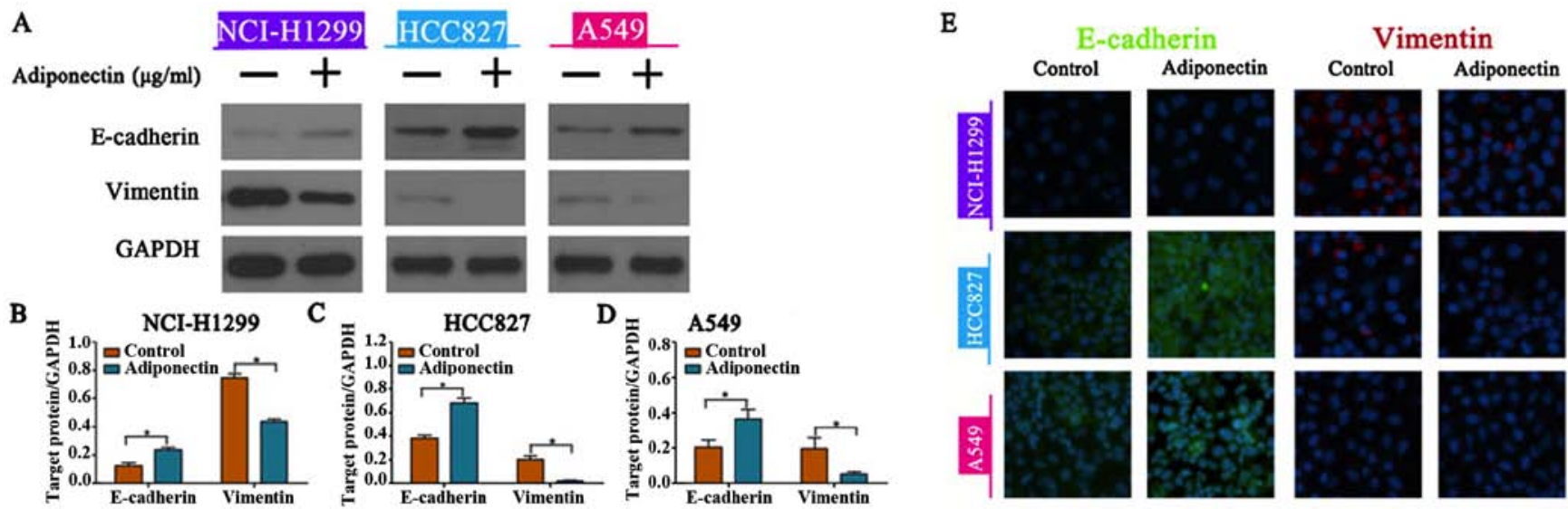

Figure 3. Adiponectin promotes E-cadherin expression and downregulates vimentin expression in NSCLC cells. (A) Western blot analysis demonstrating the expression levels of E-cadherin and vimentin following incubation of cells with adiponectin. (A-D) Exogenous adiponectin promoted overexpression of E-cadherin and underexpression of vimentin in NSCLC cells as revealed by western blotting. GAPDH served as a loading control, "P<0.05, $\mathrm{n}=3$ (each experiment). (E) Confocal microscopy images confirming the expression levels of E-cadherin and vimentin, $\mathrm{n}=3$ (each experiment).

A
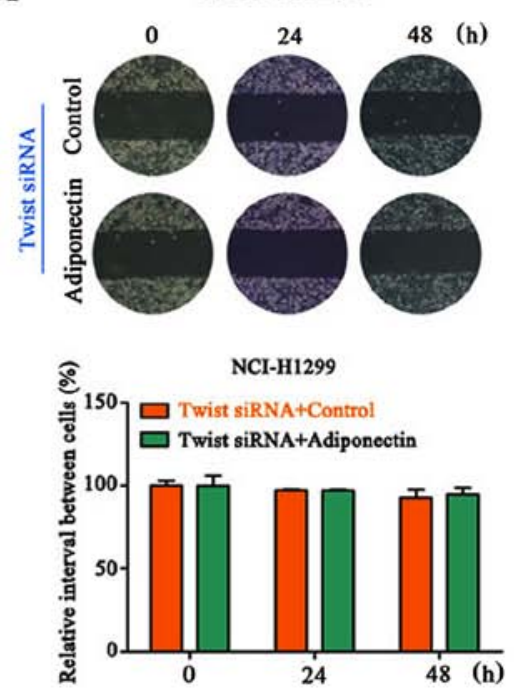

B
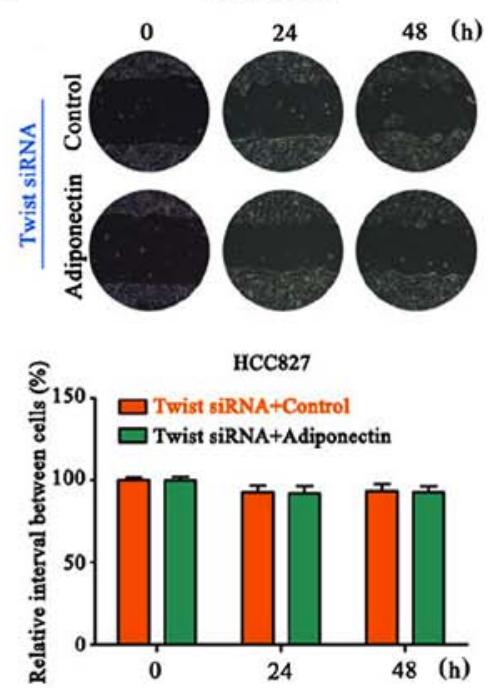

C A549
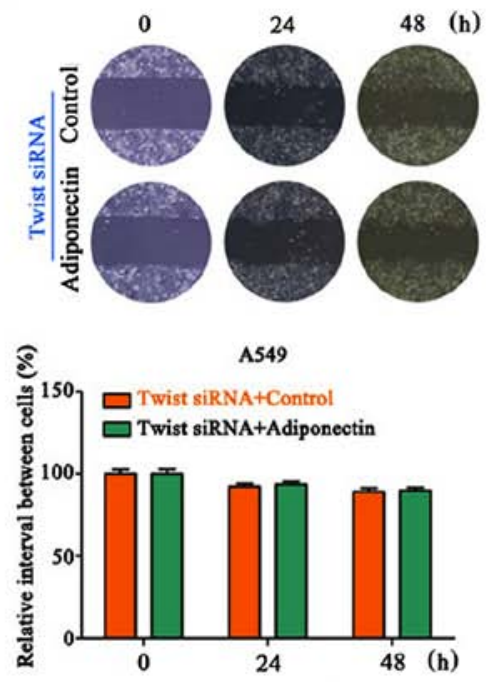
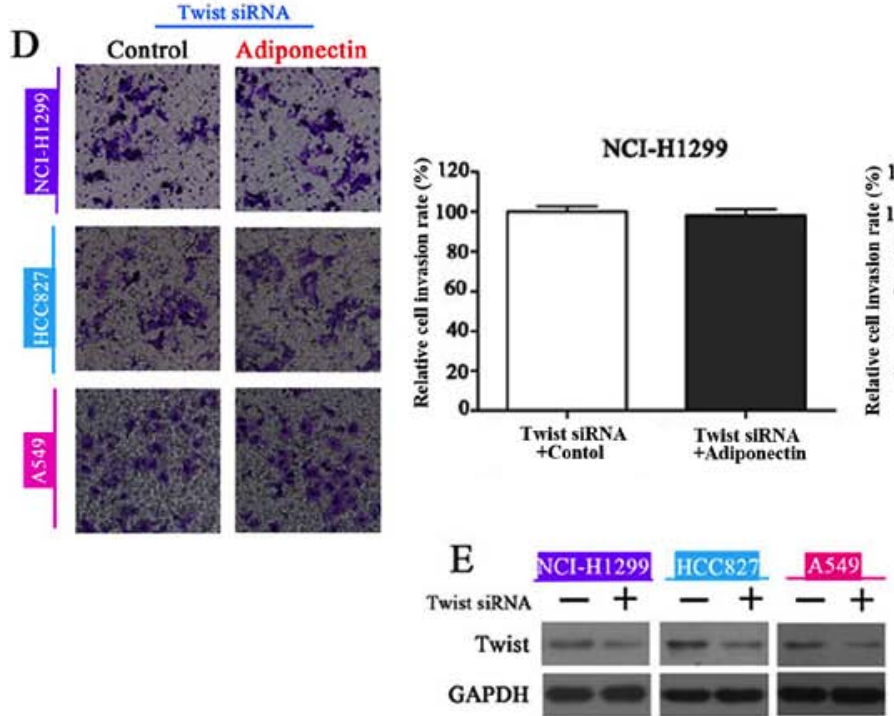
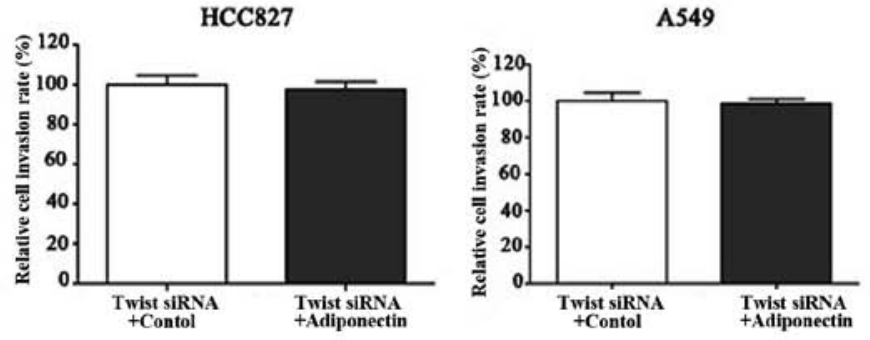

Figure 4. Twist knockdown reverses the inhibitory effects of adiponectin on NSCLC cell migration and invasion. (A-C) Wound healing assays revealed that knockdown of Twist reversed the inhibitory effects of adiponectin on NSCLC cell migration, $n=3$ (each experiment). (D) Similar effects were observed in the invasions assay, $\mathrm{n}=3$ (each experiment). (E) Twist knockdown efficiency ascertained by western blotting, ${ }^{\mathrm{P}} \mathrm{P}<0.05, \mathrm{n}=3$ (each experiment). 

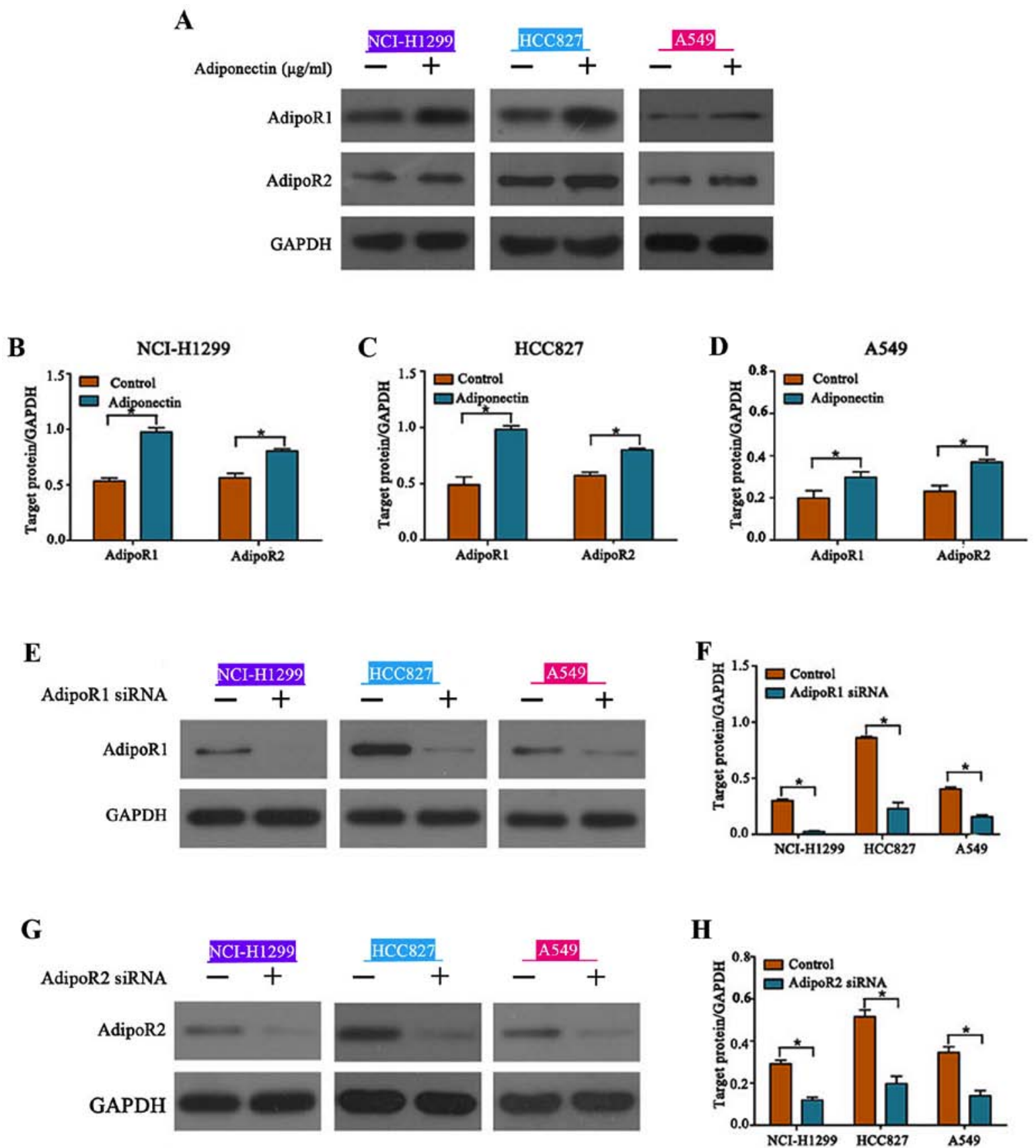

Figure 5. Adiponectin upregulates AdipoR1 and AdipoR2 expression. (A-D) Expression of AdipoR1 and AdipoR2 is significantly upregulated following adiponectin administration, ${ }^{\mathrm{P}} \mathrm{P}<0.05, \mathrm{n}=3$ (each experiment). ( $\left.\mathrm{E}-\mathrm{H}\right)$ siRNA knockdown of AdipoR1 and AdipoR2, ${ }^{*} \mathrm{P}<0.05, \mathrm{n}=3$ (each experiment).

receptors AdipoR1 and AdipoR2 and adiponectin are linked to multiple cancer-related signaling pathways including the AMPK and JNK pathways (13). We determined that cells treated with adiponectin demonstrated markedly increased expression of AdipoR1 and AdipoR2 when compared to the controls (Fig. 5A-D). To explore the role of AdipoR1 and AdipoR2 in adiponectin-mediated regulation of EMT, siRNAs were transfected to knockdown AdipoR1 and AdipoR2 expression, and knockdown efficiency was ascertained by western blotting (Fig. 5E-H). As anticipated, both AdipoR1 or AdipoR2 knockdown eliminated the inhibitory effects of adiponectin on EMT in NSCLC cells (Fig. 5I-P). These results revealed that AdipoR1 and AdipoR2 mediate the adiponectin-associated signaling pathways to regulate EMT.

\section{Discussion}

Lung metastasis and metastasis to other organs are the primary cause of NSCLC-related deaths. Accumulating evidence suggests that genetic mutations and components of the tumor microenvironment coordinately promote NSCLC metastasis $(8,9)$. Furthermore, signals from the tumor microenvironment may increase NSCLC resistance to therapies targeting genetic mutations (10). Therefore, it is essential to search for novel therapeutic targets and improve outcomes of current therapies to improve the prognosis of patients with NSCLC.

Adiponectin is the most abundant adipokine released by adipose tissues and is considered to be a significant negative 


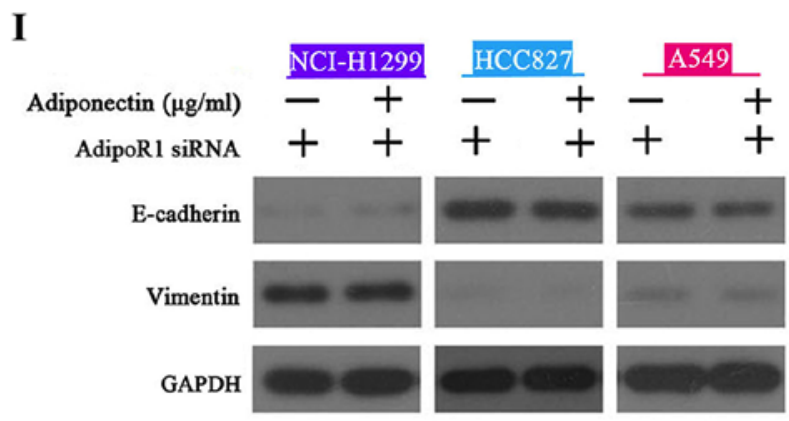

$\mathbf{J}$

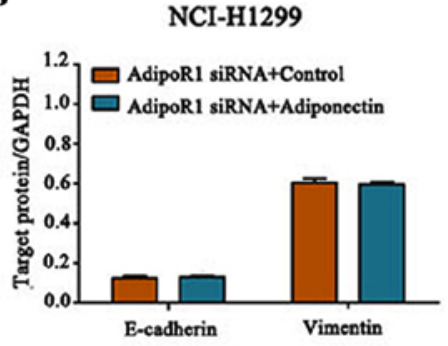

K

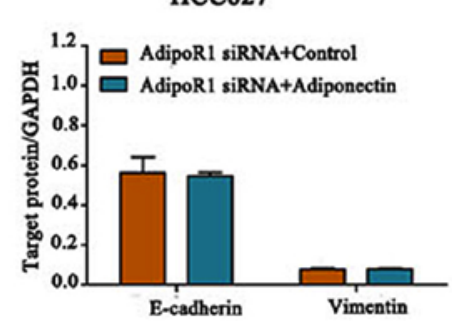

$\mathbf{L}$

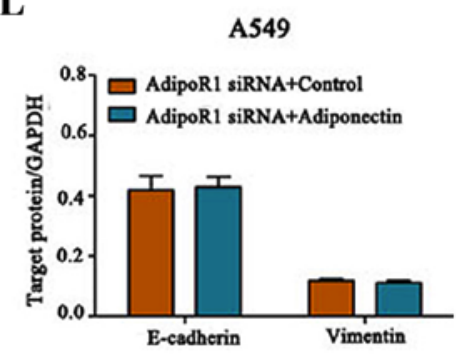

M

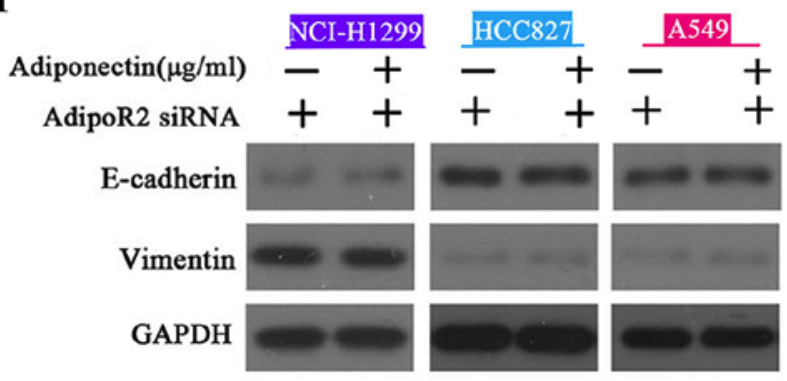

$\mathbf{N}$

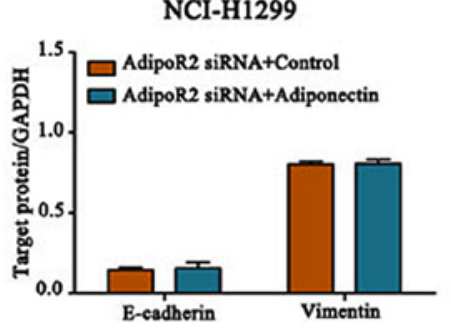

$\mathbf{O}$

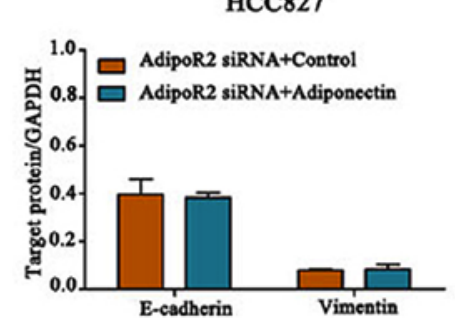

$\mathbf{P}$

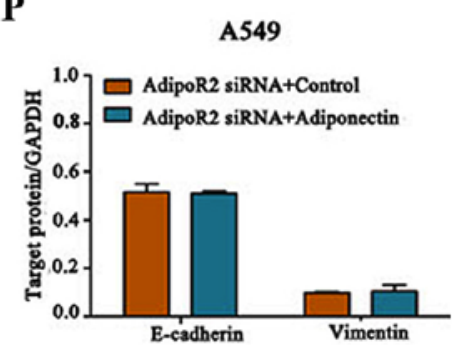

Figure 5. Continued. Adiponectin upregulates AdipoR1 and AdipoR2 expression. (I-L) AdipoR1 and (M-P) AdipoR2 knockdown reversed the inhibitory effects of adiponectin on EMT, $n=3$ (each experiment).

regulator of tumor progression, rendering it a promising potential antitumor therapeutic agent $(17,18)$. Furthermore, previous studies have confirmed that low circulating adiponectin levels are correlated with the initiation and progression of a variety of malignancies, suggesting that plasma adiponectin concentrations may serve as a biomarker for the diagnosis of malignancies $(19,20)$. Previously published studies have documented that adiponectin administration significantly inhibits tumor cell proliferation and invasion, while increasing apoptosis mainly by mediating the activation of multiple components activating the pAMPK/mTOR pathways $(21,22)$.
In contrast, other recent studies have presented a contradictory biological role for adiponectin in tumor progression. Landskroner-Eiger et al (23) revealed that adiponectin knockout not only reduced the rate of tumor growth and angiogenesis at early stages, but also promoted tumor progression in advanced malignant stages in the mouse mammary tumor virus (MMTV)-polyoma middle T antigen (PyMT) mammary tumor model. Denzel et al (24) revealed that adiponectin deficiency significantly inhibited tumor vascularization and increased apoptosis and hypoxia, while adiponectin null mice presented more pulmonary metastases. A growing body 
of evidence explains how adiponectin could exert negative biological effects on cancer. Besides enhancing angiogenesis by increasing ceramidase activity, Holland et al (25) revealed that adiponectin upregulated sphingosine-1-phosphate (S1P) expression, resulting in increased resistance to apoptosis via AdipoR1 and AdipoR2, a mechanism independent of the AMPK pathways. Adiponectin exerts different effects through interactions with other adipokines, such as increasing insulin sensitivity to promote tumor survival or neutralizing leptin to affect cellular behavior. Genetic variations in adipokine genes were revealed to be correlated with various tumors and associated with NSCLC susceptibility and development $(26,27)$. NSCLC patients exhibited a high proportion of the SNP rs2241766 in the adiponectin structural gene ADIPOQ (28).

To clarify the effects of adiponectin in NSCLC, we investigated the role of adiponectin on migration and invasion of NSCLC in vitro, and determined that adiponectin administration significantly reduced the migration and invasion of NSCLC cells. We used an approach involving western blotting and confocal microscopy to establish that adiponectin administration promoted overexpression of E-cadherin and underexpression of vimentin. In addition, Twist knockdown eliminated the inhibitory effects of adiponectin on NSCLC cell lines. These results revealed that adiponectin inhibited the ability of migration and invasion by reversing EMT in NSCLC.

Notably, in vivo studies have confirmed that low adiponectin concentrations are associated with various adverse tumor-related characteristics, but no correlation between adiponectin levels and survival outcomes of patients with malignancy has been revealed to date (29). Various factors including genetic and inflammatory parameters, hormones, percentage of body fat and body mass index regulate the production and secretion of adiponectin. Patients with advanced NSCLC commonly present with cachexia caused by cancer-related metabolic disturbances and malabsorption, which triggers a chronic systemic inflammatory state $(30,31)$. Elevated plasma adiponectin levels are considered to result from the response to inflammation, insulin resistance and adipose wastage. These observations indicated that the role of plasma adiponectin concentrations in predicting the prognosis of patients with NSCLC should be further investigated in a large cohort.

AdipoR1 and AdipoR2 are expressed in macrophages, dendritic cells (DCs) and lymphocytes, which suggests that adiponectin modulates tumor development by regulating the immune system. Sun et al (32) revealed that adiponectin deficiencies promoted tumor progression not by regulating tumor cell mitosis, vascularization and apoptosis but by reducing infiltration of macrophages in tumor tissues (32). It is well established that tumor cells can induce tumor associated macrophages (TAMs) expressing immunosuppressive chemokines and/or cytokines, and that this process is regulated by multiple factors including tumor-specific antigen expression, cytokines and other immune cells. Peng et al (33) revealed that exogenous adiponectin promoted a change from the M2 to $\mathrm{M} 1$ phenotype, characterized by upregulated expression of MHC-II, iNOS and TNF- $\alpha$ to reduce the accumulation of TAMs and tumor size. Dendritic cell signals promoting tolerance are crucial for immune escape in cancer. Tan et al (34) revealed that high expression of AdipoR1 and AdipoR2 in cancer cells was sufficient to blunt antitumor immunity by blocking activation of NF-kB in DCs. Furthermore, adiponectin inhibited inflammation by decreasing IL-17 production of $\mathrm{CD}^{+}$or $\mathrm{CD}^{+} \mathrm{T}$ lymphocytes (35). Collectively, these data demonstrated that adiponectin limited tumor development by inhibiting the activation of tumor-promoting immune cells, although it was not clear whether inhibiting antitumor immune activity similarly contributes to the effects. In the present study, we found that exogenous adiponectin increased the expression of AdipoR1 and AdipoR2, which directly inhibited migration and invasion in NSCLC. This process involved adiponectin-mediated inhibition of EMT, but may also have decreased the immune response to cancer. Adiponectin appears to have an inhibitory effect on both tumor cells and the immune response, rendering it a promising target against NSCLC. The approach for an application to clinical practice requires further investigation.

In conclusion, exogenous adiponectin not only significantly impaired NSCLC cell migration and invasion, but also reversed the EMT process. Further experiments are warranted to demonstrate and explore whether adiponectin has the same effect on NSCLC in vivo, to ultimately provide a reference for clinical treatment.

\section{Acknowledgements}

Not applicable.

\section{Funding}

The present study was financially supported by the National Natural Science Funds of China (no. 81501830), the Science Technology Department of Zhejiang Province Welfare projects (no. 3014C33245), the Zhejiang Province Natural Science Foundation of China (nos. LY16H160040 and LY15H160060), the Zhejiang Medical Scientific Research Projects (no. 2015RCB028) and co-constructed plan by the Ministry of Health and Zhejiang Province (WKJ-ZJ-1830).

\section{Availability of data and materials}

The datasets used during the present study are available from the corresponding author upon reasonable request.

\section{Authors' contributions}

XW and EC conceived the idea; HG, MS, HY and DG performed the experiments; XW, DG and WM analyzed the data; XW and EC wrote the manuscript. All authors read and approved the manuscript and agree to be accountable for all aspects of the research in ensuring that the accuracy or integrity of any part of the work are appropriately investigated and resolved.

\section{Ethics approval and consent to participate}

Not applicable.

\section{Patient consent for publication}

Not applicable. 


\section{Competing interests}

The authors declare that they have no competing interests.

\section{References}

1. Parkin DM, Bray F, Ferlay J and Pisani P: Global cancer statistics, 2002. CA Cancer J Clin 55: 74-108, 2005.

2. Chen W, Zheng R, Baade PD, Zhang S, Zeng H, Bray F, Jemal A, Yu XQ and He J: Cancer statistics in China, 2015. CA Cancer J Clin 66: 115-132, 2016.

3. Abdel-Raheem MM, Potti A, Becker WK, Saberi A, Scilley BS and Mehdi SA: Late adrenal metastasis in operable non-small-cell lung carcinoma. Am J Clin Oncol 25: 81-83, 2002.

4. National Lung Screening Trial Research Team; Aberle DR, Adams AM, Berg CD, Black WC, Clapp JD, Fagerstrom RM, Gareen IF, Gatsonis C, Marcus PM and Sicks JD: Reduced lung-cancer mortality with low-dose computed tomographic screening. N Engl J Med 365: 395-409, 2011.

5. Spira A and Ettinger DS: Multidisciplinary management of lung cancer. N Engl J Med 350: 379-392, 2004.

6. Li MJ, He Q, Li M, Luo F and Guan YS: Role of gefitinib in the targeted treatment of non-small-cell lung cancer in Chinese patients. Onco Targets Ther 9: 1291-1302, 2016.

7. Abdullah MM, Bhat A, Mohamed AK, Ching FY, Ahmed N and Gantotti S: Gefitinib as first line therapy in Malaysian patients with mutation-positive non-small-cell lung cancer: A single-center retrospective study. Oncol Lett 11: 2757-2762, 2016

8. Yu T, Guo Z, Fan H, Song J, Liu Y, Gao Z and Wang Q: Cancer-associated fibroblasts promote non-small cell lung cancer cell invasion by upregulation of glucose-regulated protein 78 (GRP78) expression in an integrated bionic microfluidic device. Oncotarget 7: 25593-25603, 2016.

9. Bertolini G, D'Amico L, Moro M, Landoni E, Perego P, Miceli R, Gatti L, Andriani F, Wong D, Caserini R,et al: Microenvironment-modulated metastatic CD133 ${ }^{+} / \mathrm{CXCR}^{+} /$ EpCAM-lung cancer-initiating cells sustain tumor dissemination and correlate with poor prognosis. Cancer Res 75: 3636-3649, 2015.

10. Kurimoto $R$, Iwasawa $S$, Ebata $T$, Ishiwata $T$, Sekine I, Tada Y, Tatsumi K, Koide S, Iwama A and Takiguchi Y: Drug resistance originating from a TGF- $\beta / F G F-2$-driven epithelial-to-mesenchymal transition and its reversion in human lung adenocarcinoma cell lines harboring an EGFR mutation. Int J Oncol 48: 1825-1836, 2016.

11. Folco EJ, Rocha VZ, López-Ilasaca M and Libby P: Adiponectin inhibits pro-inflammatory signaling in human macrophages independent of interleukin-10. J Biol Chem 284: 25569-25575, 2009.

12. Yokota T, Oritani K, Takahashi I, Ishikawa J, Matsuyama A, Ouchi N, Kihara S, Funahashi T, Tenner AJ, Tomiyama Y and Matsuzawa Y: Adiponectin, a new member of the family of soluble defense collagens, negatively regulates the growth of myelomonocytic progenitors and the functions of macrophages. Blood 96: 1723-1732, 2000.

13. Kelesidis I, Kelesidis T and Mantzoros CS: Adiponectin and cancer: A systematic review. Br J Cancer 94: 1221-1225, 2006.

14. Barb D, Williams CJ, Neuwirth AK and Mantzoros CS: Adiponectin in relation to malignancies: A review of existing basic research and clinical evidence. Am J Clin Nutrit 86 (Suppl): S858-S866, 2007.

15. Tang $\mathrm{CH}$ and Lu ME: Adiponectin increases motility of human prostate cancer cells via adipoR, p38, AMPK, and NF-kappaB pathways. Prostate 69: 1781-1789, 2009.

16. Umekawa K, Kimura T, Kudoh S, Suzumura T, Nagata M, Mitsuoka S, Matsuura K, Oka T, Yoshimura N, Kira Y and Hirata K: Reaction of plasma adiponectin level in non-small cell lung cancer patients treated with EGFR-TKIs. Osaka City Med J 59: 53-60, 2013.

17. Vansaun MN: Molecular pathways: Adiponectin and leptin signaling in cancer. Clin Cancer Res 19: 1926-1932, 2013.

18. Otani K, Kitayama J, Yasuda K, Nio Y, Iwabu M, Okudaira S, Aoki J, Yamauchi T, Kadowaki T and Nagawa H: Adiponectin suppresses tumorigenesis in $A p c^{\mathrm{Min} /+}$ mice. Cancer Lett 288: $177-182,2010$
19. Soliman PT, Wu D, Tortolero-Luna G, Schmeler KM, Slomovitz BM, Bray MS, Gershenson DM and Lu KH: Association between adiponectin, insulin resistance, and endometrial cancer. Cancer 106: 2376-2381, 2006.

20. Ishikawa M, Kitayama J, Kazama S, Hiramatsu T, Hatano K and Nagawa H: Plasma adiponectin and gastric cancer. Clin Cancer Res 11: 466-472, 2005.

21. Kim KY, Baek A, Hwang JE, Choi YA, Jeong J, Lee MS, Cho DH, Lim JS, Kim KI and Yang Y: Adiponectin-activated AMPK stimulates dephosphorylation of AKT through protein phosphatase 2A activation. Cancer Res 69: 4018-4026, 2009.

22. Barb D, Neuwirth A, Mantzoros CS and Balk SP: Adiponectin signals in prostate cancer cells through Akt to activate the mammalian target of rapamycin pathway. Endocr Relat Cancer 14: 995-1005, 2007.

23. Landskroner-Eiger S, Qian B, Muise ES, Nawrocki AR, Berger JP, Fine EJ, Koba W, Deng Y, Pollard JW and Scherer PE: Proangiogenic contribution of adiponectin toward mammary tumor growth in vivo. Clin Cancer Res 15: 3265-3276, 2009.

24. Denzel MS, Hebbard LW, Shostak G, Shapiro L, Cardiff RD and Ranscht B: Adiponectin deficiency limits tumor vascularization in the MMTV-PyV-mT mouse model of mammary cancer. Clin Cancer Res 15: 3256-3264, 2009.

25. Holland WL, Miller RA, Wang ZV, Sun K, Barth BM, Bui HH, Davis KE, Bikman BT, Halberg N, Rutkowski JM, et al: Receptor-mediated activation of ceramidase activity initiates the pleiotropic actions of adiponectin. Nat Med 17: 55-63, 2011.

26. Ribeiro R, Araújo AP, Coelho A, Catarino R, Pinto D, Araújo A, Calçada C, Lopes C and Medeiros R: A functional polymorphism in the promoter region of leptin gene increases susceptibility for non-small cell lung cancer. Eur J Cancer 42: 1188-1193, 2006.

27. Shih CM, Lee YL, Chiou HL, Chen W, Chang GC, Chou MC and Lin LY: Association of TNF-alpha polymorphism with susceptibility to and severity of non-small cell lung cancer. Lung Cancer 52: 15-20, 2006

28. Cui E, Deng A, Wang X, Wang B, Mao W, Feng X and Hua F: The role of adiponectin (ADIPOQ) gene polymorphisms in the susceptibility and prognosis of non-small cell lung cancer. Biochem Cell Biol 89: 308-313, 2011.

29. Gioulbasanis I, Georgoulias P, Vlachostergios PJ, Baracos V, Ghosh S, Giannousi Z, Papandreou CN, Mavroudis D and Georgoulias V: Mini nutritional assessment (MNA) and biochemical markers of cachexia in metastatic lung cancer patients: Interrelations and associations with prognosis. Lung Cancer 74: 516-520, 2011.

30. Bruera E: ABC of palliative care. Anorexia, cachexia, and nutrition. BMJ 315: 1219-1222, 1997.

31. Batista ML Jr, Olivan M, Alcantara PS, Sandoval R, Peres SB, Neves RX, Silverio R, Maximiano LF, Otoch JP and Seelaender M: Adipose tissue-derived factors as potential biomarkers in cachectic cancer patients. Cytokine 61: 532-539, 2013.

32. Sun Y and Lodish HF: Adiponectin deficiency promotes tumor growth in mice by reducing macrophage infiltration. PloS One 5: e11987, 2010.

33. Peng J, Tsang JY, Ho DH, Zhang R, Xiao H, Li D, Zhu J, Wang F, Bian Z, Lui VC, et al: Modulatory effects of adiponectin on the polarization of tumor-associated macrophages. Int J Cancer 137: 848-858, 2015

34. Tan PH, Tyrrell HE, Gao L, Xu D, Quan J, Gill D, Rai L, Ding Y, Plant G, Chen Y, et al: Adiponectin receptor signaling on dendritic cells blunts antitumor immunity. Cancer Res 74: 5711-5722, 2014

35. Shibata S, Tada Y, Hau CS, Mitsui A, Kamata M, Asano Y, Sugaya M, Kadono T, Masamoto Y, Kurokawa M, et al: Adiponectin regulates psoriasiform skin inflammation by suppressing IL-17 production from gammadelta-T cells. Nat Commun 6: 7687, 2015.

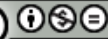

This work is licensed under a Creative Commons Attribution-NonCommercial-NoDerivatives 4.0 International (CC BY-NC-ND 4.0) License. 